\title{
Angular Independence of Break Position for Magnetic Power Spectral Density in Solar Wind Turbulence
}

\author{
Die Duan ${ }^{1}$, Jiansen $\mathrm{He}^{1}$ (D), Zhongtian $\mathrm{Pei}^{1}$, Shiyong Huang ${ }^{2}$, Honghong Wu ${ }^{1,3}$, Daniel Verscharen ${ }^{3,4}$ (iD), and Linghua Wang ${ }^{1}$ (D) \\ ${ }^{1}$ School of Earth and Space Sciences, Peking University, Beijing 100871, People's Republic of China; jshept@ pku.edu.cn \\ ${ }^{2}$ Electronic Information School, Wuhan University, Wuhan 430079, People's Republic of China \\ ${ }^{3}$ Mullard Space Science Laboratory, University College London, Dorking RH5 6NT, UK \\ ${ }^{4}$ Space Science Center, University of New Hampshire, Durham, NH 03824, USA \\ Received 2018 March 4; revised 2018 June 16; accepted 2018 June 21; published 2018 September 25
}

\begin{abstract}
The break in power spectral density (PSD) around the ion scales indicates the onset of dissipation and/or dispersion of kinetic turbulence. For Alfvén waves in the kinetic regime, the dissipation and dispersion are individually dependent on the propagation angle, $\theta_{\mathrm{kB}}$, which has $\theta_{\mathrm{RB}}$ (the angle between radial direction and local mean magnetic field direction) as a proxy in solar wind measurements. The relation between $\theta_{\mathrm{RB}}$ and the break position helps us find the role of dissipation and/or dispersion for deforming the PSD profile. In order to locate the spectral break position automatically and quantitatively, we develop a dual-power-law fitting method to fit the PSD profiles in both MHD and kinetic ranges simultaneously. The break position $f_{\mathrm{b}}$ is found to change little with $\theta_{\mathrm{RB}}$, suggesting an angular independence of the spectral break. Furthermore, $f_{\mathrm{b}}$ in our statistical study of fast solar wind near $1 \mathrm{au}$ is consistent with a wavenumber $k$ satisfying $k\left(\rho_{\mathrm{p}}+d_{\mathrm{p}}\right) \sim 1\left(\rho_{\mathrm{p}}\right.$ is the thermal proton gyroradius and $d_{\mathrm{p}}$ is the proton inertial length), independently of $\theta_{\mathrm{RB}}$. To interpret this independence, we incorporate the effects of both dissipation and dispersion in a unified description, which is the breakdown of the magnetic frozen-in condition in wavenumber space $\left(k_{\|}, k_{\perp}\right)$. The breakdown of the frozen-in condition is relatively isotropic compared to the strong anisotropy of dispersion and dissipation. Furthermore, the spatial scale for the onset of the breakdown frozen-in condition is estimated to be the sum of $\rho_{\mathrm{p}}$ and $d_{\mathrm{p}}$.
\end{abstract}

Key words: solar wind - turbulence

\section{Introduction}

The Solar wind in the heliosphere is often regarded as a natural laboratory for collisionless plasma turbulence. The Omnidirectional (or reduced) power spectral density (PSD) of the turbulent fluctuations is divided into three parts in wavenumber space: an energy-containing range at small wavenumbers, an inertial range of cascading energy at medium wavenumbers, and a dissipation range at large wavenumbers (Tu \& Marsch 1995; Bruno \& Carbone 2013; Kiyani et al. 2015). Different power-law functions can be used to describe the energy-containing range and inertial range of the turbulence PSD in the solar wind. For example, the PSD of the magnetic turbulence in the energy-containing range and the inertial range can be approximated by power-law functions with a power index of -1 and $-5 / 3$, respectively. At smaller scales beyond the inertial range, turbulent energy begins to dissipate and is transferred to the thermal energy of the plasma while wave behavior becomes dispersive. The onset of the dissipation and/or dispersion causes the power-law spectral profile to be broken at the corresponding scale, leading to a steeper segment of the magnetic PSD beyond the inertial range. It is found that the magnetic PSD between the ion kinetic scale and the electron kinetic scale can be approximated by a third powerlaw function with a power index between -2 and -4 (Leamon et al. 1998; Alexandrova et al. 2009; Sahraoui et al. 2009; Smith et al. 2012). This range is called a secondary inertial range or electron inertial range, where the electron behavior can still be approximated through a fluid description (Biskamp et al. 1996; Li et al. 2001; Galtier \& Bhattacharjee 2003). At scales close to or even smaller than the electron kinetic scales, there is a dispute regarding whether the turbulence PSD is another steeper power-law function or a stretched exponential function (Sahraoui et al. 2010; Alexandrova et al. 2012).

The spectral break between the inertial range and the dissipation range is often interpreted as the onset of dissipation. On the other hand, the spectral break may be related to the transition to a dispersive behavior of the associated modes (around $k \rho_{\mathrm{p}} \sim 1$ ). Both dissipation and dispersion can give rise to the break; however, it is difficult to determine the contribution of these two mechanisms to the position and strength of the break. For plasma turbulence consisting of kinetic waves, the dispersive behavior of kinetic waves will influence both the magnetic and electric PSDs. For example, the dissipation range of magnetic PSD with kinetic Alfvén turbulence and whistler turbulence are steeper than their MHD counterpart, with the power spectral index predicted to be around $-7 / 3$ and -2.5 , respectively (Biskamp et al. 1999; Galtier 2006; Schekochihin et al. 2009; Narita \& Gary 2010; Zhao et al. 2016). The electric field fluctuations are enhanced at smaller scales, leading to a shallower PSD with a spectral index predicted to be about $-1 / 3$ and $-1 / 2$. Such different behaviors of magnetic and electric PSDs have been observed in solar wind and magnetosheath kinetic turbulence, suggesting the role of dispersive behavior in altering the polarization relationship between magnetic and electric field fluctuations and the consequent scaling difference between the magnetic and electric PSDs (Bale et al. 2005; Matteini et al. 2017). The transition from a linear-in- $\mathrm{k}$ to a nonlinear-in-k dispersion relation from the MHD to the kinetic regime also depends on various plasma parameters, e.g., the proton and electron plasma beta, the electron to proton temperature ratio, and so on (Boldyrev et al. 2015). However, it is still a challenge for the 
field to distinguish the role of dispersion in shaping the PSD profile from the effect of dissipation.

Solar wind turbulence, especially in fast streams, is anisotropic in both scaling and the level of the reduced PSDs, which are obtained by sampling the turbulence in different directions with respect to local background magnetic field (Horbury et al. 2008; Podesta 2009; Luo \& Wu 2010). The nature of the reduced PSD is still unclear, e.g., the existence of the "slab + 2D" distribution (Matthaeus et al. 1990; Bieber et al. 1996) or the "pure critical balance" distribution of power in wavevector space (Goldreich \& Sridhar 1995). Some endeavors have been made to reconstruct the multidimensional PSD in wavevector space by applying the tomography method based on the projection-slice theorem to single-spacecraft data (He et al. 2013) or the k-filtering wave-telescope technique to four-spacecraft measurements from Cluster (Sahraoui et al. 2010; Narita et al. 2011). A magnetic PSD distribution of "slab + oblique ridge" is found in fast solar wind streams the inner heliosphere between 0.3 and 1 au (He et al. 2013). At ion kinetic scales just beyond the MHD regime, two types of kinetic waves are found to be consistent with the two components of the magnetic helicity angular spectrum, where quasi-perpendicular kinetic Alfvén waves and quasi-parallel ion-cyclotron waves are the major and minor wave populations, respectively (He et al. 2011, 2012a, 2012b; Telloni et al. 2012; Roberts \& Li 2015). Therefore, there are angular dependencies in both the PSD in the MHD regime and the wave-mode nature in the ion kinetic regime. Moreover, the cascaded energy flux is anisotropic with the perpendicular rate greater than the parallel cascade rate, especially when the solar wind is noncompressive (MacBride et al. 2008). This anisotropy is weaker when the solar wind is more compressive (Hadid et al. 2017). However, the angular dependence of the spectral break between the MHD scales and ion/sub-ion kinetic scales is still unknown, and the answer to this question will cast beneficial insight into the onset process of dissipation and/or dispersion of kinetic turbulence.

The radial evolution and $\beta$-dependence of the spectral break position have recently been investigated. Using the magnetic field measurements from the Ulysses and Messenger spacecraft, Perri et al. (2010) studied the radial evolution of the spectral break near the ion scale, but did not find a significant decrease of break frequency along with the increasing heliocentric distance. This result is consistent with a later study based on Helios data between 0.3 and 0.9 au (Bourouaine et al. 2012). It was proposed that the spatial scale corresponding to the break frequency seems to follow the proton inertial length $\left(d_{\mathrm{p}}\right)$, when taking into account the twodimensional nature of the magnetic fluctuations and assuming the wavevector $\boldsymbol{k}$ to be in the plane of $\boldsymbol{V}_{\mathrm{sw}}$ (solar wind velocity) and $\boldsymbol{B}_{\mathrm{bg}}$ (background magnetic field). However, as pointed out by Bruno \& Trenchi (2014), the Messenger spacecraft did not provide solar wind plasma data and was thus unable to determine if the solar wind conditions encountered by Messenger were of the same type as those measured by Ulysses. To make the analysis result more credible, Bruno \& Trenchi (2014) chose intervals when the Messenger, WIND, and Ulysses were almost aligned in the radial direction, and then analyzed the magnetic PSD at different locations. As a result, they found that the spectral break frequency reduces significantly with increasing distance. Moreover, the trend is consistent with that of the scale corresponding to proton cyclotron resonance, if the isotropy assumption is imposed on the proton thermal state. However, invoking proton cyclotron damping to explain the spectral break cannot cover the whole picture, since quasi-parallel Alfvén cyclotron waves only contribute a minor part to the reduced PSD in observations.

Ion plasma $\beta_{\mathrm{p}}$, which is a measure of the ratio between ion thermal pressure $\left(P_{\mathrm{th}, \mathrm{p}}\right)$ and magnetic pressure $\left(P_{\mathrm{B}}\right)$, is also the squared ratio of the two ion scales $\left(\beta_{\mathrm{p}}=\left(\rho_{\mathrm{p}} / d_{\mathrm{p}}\right)^{2}\right)$. Therefore it is also interesting to see the behavior of the spectral break position $\left(f_{\mathrm{b}}\right)$ as a function of $\beta_{\mathrm{p}}$. It is found that $f_{\mathrm{b}}$ approaches $f_{\rho_{\mathrm{p}}}$ at very large $\beta_{\mathrm{p}}$ and approaches $f_{\mathrm{d}_{\mathrm{p}}}$ at very small $\beta_{\mathrm{p}}$, where $f_{\rho_{\mathrm{p}}}=V_{\mathrm{sw}} /\left(2 \pi \rho_{p}\right)$ and $f_{\mathrm{d}_{\mathrm{p}}}=V_{\mathrm{sw}} /\left(2 \pi d_{p}\right)$ is the frequency in the spacecraft frame corresponding to the spatial scales satisfying $k \rho_{\mathrm{p}}=1$ and $k d_{\mathrm{p}}=1$, respectively (Chen et al. 2014). In the range with $\beta$ between 0.1 and $1.3, f_{\mathrm{b}} / f_{\mathrm{d}_{\mathrm{p}}}$ is found to be of order 0.5 , and $f_{\mathrm{b}} / f_{\rho_{\mathrm{p}}}$ increases from 0.2 to 0.5 (Wang et al. 2018). In $\beta_{\mathrm{p}, \perp} \sim 1$ plasma, the break is reported to be associated with the proton cyclotron resonance scale, $k_{\mathrm{c}}=1 /\left(\rho_{\mathrm{p}}+d_{\mathrm{p}}\right)$ (Woodham et al. 2018). The analysis by Smith et al. (2012) for $\beta_{\mathrm{p}}>1$ solar winds shows no dependence of the spatial spectral break scale on $\theta_{\mathrm{RB}}$. The $f_{\mathrm{b}}$ position cannot be solely attributed to the dispersion relation of kinetic Alfvén waves, especially in cases with $\beta_{i} \ll 1$ and $\beta_{\mathrm{e}} \ll 1$. The dissipation of waves/turbulence should be taken into account when considering the physical process underlying the spectral break. In 2D hybrid simulations, the spatial scale ratios between the spectral break scale $\left(l_{\mathrm{b}}\right)$ and proton characteristic scales, $l_{\mathrm{b}} / d_{\mathrm{p}}$ and $l_{\mathrm{b}} / \rho_{\mathrm{p}}$, are found to have an increasing and a decreasing trend with increasing $\beta_{\mathrm{p}}$, respectively (Franci et al. 2016). The spectral break scale $l_{\mathrm{b}}$ in simulations is smaller than in observations, which means that the onset of dispersion/dissipation appears at shorter scales in the simulation. Such a difference indicates the complexity of real solar wind turbulence, which cannot be fully reproduced by $2 \mathrm{D}$ hybrid simulations. Furthermore, in 2D hybrid simulations, the PSD of density fluctuations is less than the PSD of fluctuations in the normalized parallel magnetic-field component at low $\beta \sim 0.2$, while the two are comparable to one another at intermediate and higher $\beta$ (Cerri et al. 2016). These features suggest the dominance of whistler dynamics over KAW dynamics in the regime of lower $\beta$, and a switch to the opposite regime at intermediate and higher $\beta$ (Cerri et al. 2016).

Whether the spectral break position $\left(f_{\mathrm{b}}\right)$ changes with the angle between the radial direction and the local mean magnetic field direction $\left(\theta_{\mathrm{RB}}\right)$ is the aim of this work. The spectral break positions of the quasi-parallel and quasi-perpendicular PSDs can be distinguished in this way, which is helpful for the anisotropy diagnosis of underlying dissipation or/and dispersion mechanisms. In Section 2, we propose a novel method to fit the dual-power-law PSD profiles with a composite function in one step and automatically locate the transition position of the dual-power-law profiles. We then apply this method to a case of solar wind turbulence, illustrating the success of this method in fitting the dual-power-law PSDs and locating the spectral break positions at different $\theta_{\mathrm{RB}}$. In Section 3, a statistical study based on 20 events is conducted, the result of which indicates the angular independence (isotropy) of $f_{\mathrm{b}}$. Section 4 is reserved for summary and discussion.

\section{A Method to Fit the Dual-power-law Spectral Profile and to Locate the Spectral Break Position}

To locate the position of the spectral break, the first step is to find a function that can fit the dual-power-law PSD profile well. 
We propose a function as a sum of two modified power laws:

$$
\begin{gathered}
P S D(f)=A_{1} f^{-\alpha_{1}} g_{1}(f)+A_{2} f^{-\alpha_{2}} g_{2}(f), \\
g_{1}(f)=e^{-f^{3} p}, \\
g_{2}(f)=e^{-\frac{1}{f^{3} p},}
\end{gathered}
$$

where $A_{n} f^{-\alpha_{n}}(n=1,2)$ represent the two power functions. The subscript $n=1$ is for the inertial range, and $n=2$ is for the dissipation range. $A_{n}$ relates to the level of the spectra, and $\alpha_{n}$ is the spectral index. The parameter $p$ is another fitting parameter that determines the position of the break $\left(f_{\mathrm{b}}\right) . g_{1}(f)$ and $g_{2}(f)$ are two auxiliary functions that control the dominance of the two power functions in two different frequency ranges, respectively. $g_{1}(f)$ is close to 1 over a frequency lower than $f_{\mathrm{b}}$ and approaches 0 in higher frequency ranges beyond $f_{\mathrm{b}} . g_{2}(f)$ shows the opposite behavior. These two functions help to shape the fitting function and generate different spectral indices in different frequency ranges. For example, in the inertial range, the second term of the function is negligible, and the PSD profile is mainly described by the first term. In the vicinity of the break, the two terms have the same order of magnitude, which make the profile of the fitting function curved.

To get the position of the break, the curvature at every discrete frequency point of the fitting line is calculated. The formula of the curvature reads

$$
\kappa=\frac{\left|\log (\mathrm{PSD})^{\prime \prime}\right|}{\left(1+\log (\mathrm{PSD})^{\prime 2}\right)^{\frac{3}{2}}},
$$

where $\log (\mathrm{PSD})^{\prime}=\Delta \log (\mathrm{PSD}) / \Delta \log (f)$ and $\log (\mathrm{PSD})^{\prime \prime}=$ $\Delta \log (\mathrm{PSD})^{\prime} / \Delta \log (f)$ are the first- and second-order derivatives in logarithmic coordinates, respectively. Curvature for a straight line equals 0 and becomes larger when the line is more curved. The break point of the PSD is supposed to have the maximum curvature, where the two power-law functions connect. Therefore, the point with the maximum curvature is selected as the break point. The uncertainty of the identified break position is estimated with the standard deviation of a sequence of $f_{\mathrm{b}, \mathrm{i}}(i=1, \ldots, N)$, which are obtained by fitting and locating the PSD profiles $\mathrm{N}$ times with the discrete frequencies being randomly resampled $\mathrm{N}$ times. We set the value of $\mathrm{N}$ to 200. At every step, two points in the frequency domain are randomly chosen and dropped, leaving the PSDs at the other frequencies to be fitted. This method to estimate the $f_{\mathrm{b}}$ uncertainty is called "bootstrap" method in statistics. We note that a modest change of the power index in $g_{1}(f)$ and $g_{2}(f)$ does not affect the estimated position of the break point.

\section{Applying the Dual-power-law Fitting Method to a Case of Fast Solar Wind}

We use the in situ magnetic field measurements obtained from the WIND spacecraft, which is orbiting the Sun around the solar-terrestrial Lagrangian $L_{1}$ point. The magnetic field data was sampled at a time cadence of $0.092 \mathrm{~s}$ by MFI (Lepping et al. 1995). We select 20 cases of high-speed solar wind streams between 1995 and 1996 for a statistical study of the angular dependence of the spectral break position. Our selection is based on the following criteria: (1) flow speed is greater than $550 \mathrm{~km} \mathrm{~s}^{-1}$; (2) density and magnetic field strength is stable $(\delta n /\langle n\rangle,|\delta B| /\langle|B|\rangle<0.2)$ for more than $24 \mathrm{hr}$; (3) proton plasma $\beta$ is between 0.7 and 1.3 ; and (4) no ICME event is embedded fully or partially within the time intervals. These criteria guarantee that potential common features stand out to be discerned and explored by means of our statistical study.

We first choose data of a fast solar wind stream in the time range between 1996 March 12 and 13. Trace power spectral densities at 25 discrete frequencies from 0.01 to $1.4 \mathrm{~Hz}$ in the spacecraft frame are calculated with the help of a wavelet transform (Torrence \& Compo 1998). The reason for the upper limit of the frequency being set below the Nyquist frequency $(\sim 5.4 \mathrm{~Hz})$ is to avoid the contamination of the PSD from the noise floor as well as the aliasing effect at the higher frequency end. The profile of PSD as calculated from the wavelet transform without distinguishing different $\theta_{\mathrm{RB}}$ angles is plotted in Figure 1 (see the sequence of small black hollow squares in the figure). The dual-power-law shape of the PSD profile with a spectral break located in between can easily be recognized by eye.

To further quantify the PSD profile, we employ the method described in Section 2 to fit the calculated PSD. The fitting result (the dark-blue dashed line) matches well with the measured PSD. The green line and the cyan line represent the PSD profiles for the two terms in the lower and higher frequency ranges respectively. As an output of the dual-powerlaw fitting method, the spectral indexes in both the inertial range and the dissipation range are obtained simultaneously, which gives values of -1.63 and -3.16 for the example case presented here. The spectral break position as defined through the maximum curvature is labeled with a blue solid square. For comparison, three frequencies in the spacecraft frame, which correspond to three different spatial scales under Taylor's frozen-in hypothesis $\left(f_{\mathrm{SC}}=V_{\mathrm{SW}} / \lambda, \quad \lambda=2 \pi / k\right)$, are also labeled. The frequency $\left(f_{\rho}\right)$ for the spatial scale related to the proton gyroradius $\left(k \rho_{\mathrm{p}}=1\right)$ is labeled with a red solid triangle, with the proton gyroradius $\rho_{\mathrm{p}}$ being calculated with the proton temperature. The red solid pentagram represents the frequency $\left(f_{\mathrm{d}}\right)$ for the spatial scale related to the proton inertial length $\left(k d_{\mathrm{p}}=1\right)$. The frequency $\left(f_{\mathrm{c}}\right)$ for the spatial scale roughly satisfying the cyclotron resonance condition $\left(k_{\mathrm{c}}=\Omega_{\mathrm{p}} /\left(V_{\mathrm{A}}+\right.\right.$ $\left.\left.V_{\mathrm{th}}\right), k_{\mathrm{c}}\left(\rho_{\mathrm{p}}+d_{\mathrm{p}}\right)=1\right)$ (Leamon et al. 1998) is plotted as a red solid square. The data for calculation are from MFI (for magnetic field) and PESA-low/3DP (for proton density and temperature; Lin et al. 1995) on WIND. Another characteristic frequency is the proton gyrofrequency $\left(f_{\mathrm{g}}\right)$ as labeled with a red solid circle in the figure. It can be seen that $f_{\mathrm{c}}$ is closest to $f_{\mathrm{b}}$, while $f_{\mathrm{g}}$ is much lower than $f_{\mathrm{b}}$, while $f_{\rho}$ and $f_{\mathrm{d}}$ are obviously beyond $f_{\mathrm{b}}$.

As one important aspect of the turbulence anisotropy study, it is necessary to investigate if the onset of dissipation and/or dispersion is anisotropic in wavevector space. In other words, we investigate if the spectral break position of the reduced PSD depends on and changes with $\theta_{\mathrm{RB}}$, the angle between the local mean magnetic field direction and the radial direction (approximated to sampling direction around and beyond 


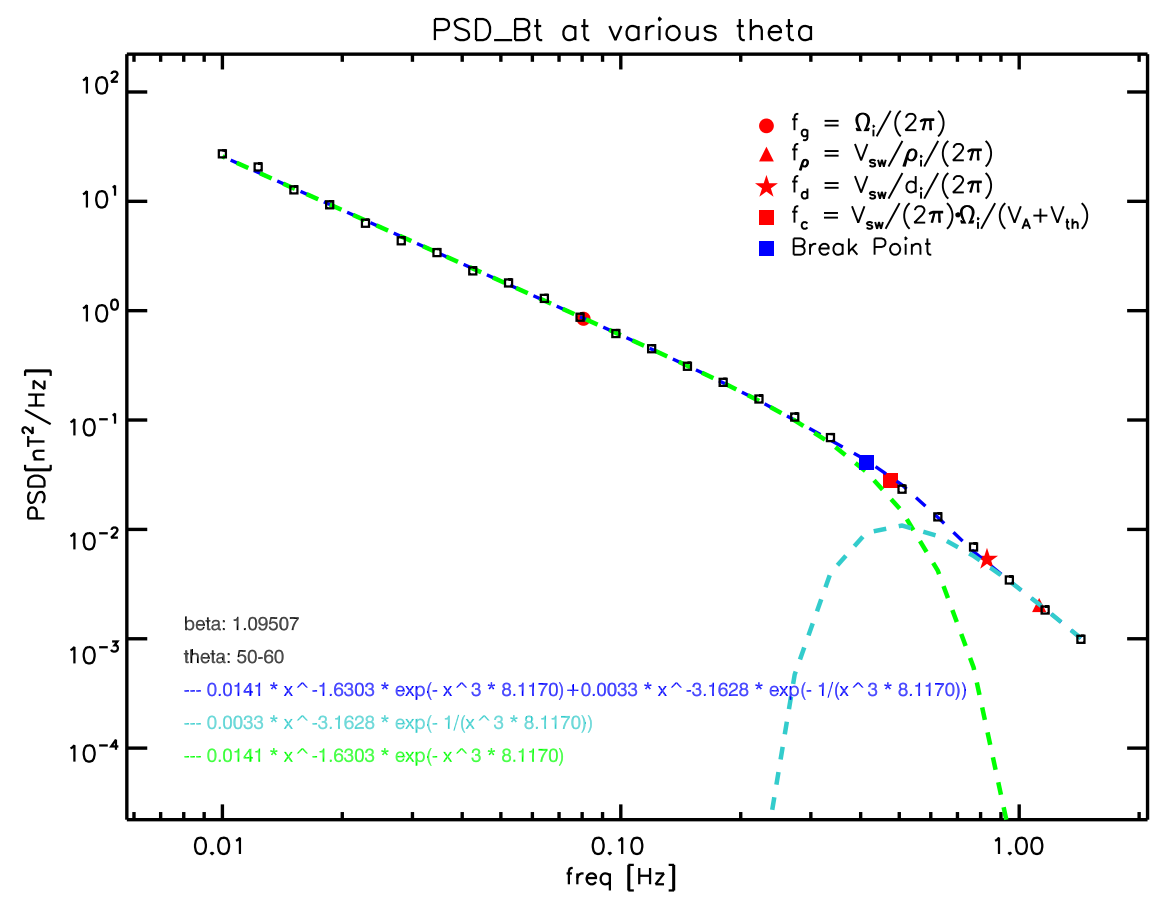

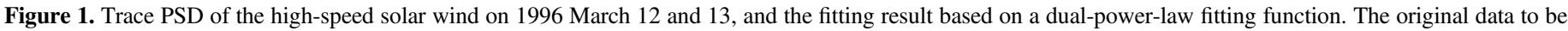

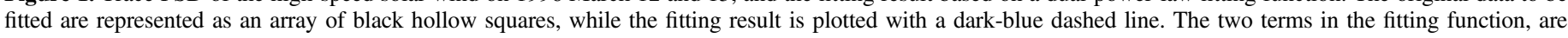

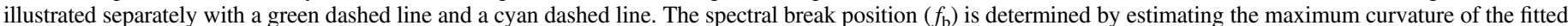

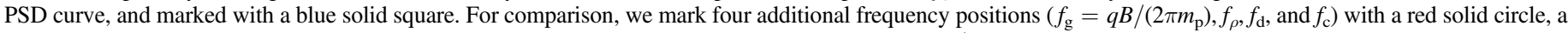

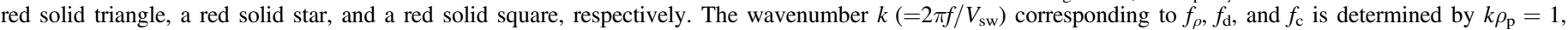
$k d_{\mathrm{p}}=1, k\left(\rho_{\mathrm{p}}+d_{\mathrm{p}}\right)=1$, respectively. It can be seen that $f_{\mathrm{b}}$ is closer to $f_{\mathrm{c}}$ than to any of the other characteristic frequencies.

$1 \mathrm{au})$. For this objective, we plot and fit the PSD profiles at different $\theta_{\mathrm{RB}}$. and thereby automatically obtain the spectral break positions of the PSD profiles.

We group the wavelet spectra of the magnetic field fluctuations at each period $(\delta \tilde{\boldsymbol{B}}(t, p))$ into nine bins according to the their time-dependent local $\theta_{\mathrm{RB}}(t, p)$ at the corresponding period $p$. Each bin contains data with the corresponding $\theta_{\mathrm{RB}}$ located within $10^{\circ}$. For instance, the first bin contains the data with $\theta_{\mathrm{RB}}$ from $0^{\circ}$ to $10^{\circ}$, and the last bin for $\theta_{\mathrm{RB}}$ from $80^{\circ}$ to $90^{\circ}$. The nine PSDs of the nine $\theta_{\mathrm{RB}}$ bins are constructed from the redistributed wavelet spectra. The nine PSD profiles are plotted in Figure 2. The profile at the bottom is for the bin of $\theta_{\mathrm{RB}}$ between $0^{\circ}$ and $10^{\circ}$, and the top one for the bin of $\theta_{\mathrm{RB}}$ between $80^{\circ}$ and $90^{\circ}$. To compare the PSDs with different angles in one figure, the $n$th PSD profile is offset with its level being multiplied by $10^{n}$. It can be seen that the quasi-perpendicular spectra are stronger than the quasi-parallel spectra. We note that $\theta_{\mathrm{RB}}$ is calculated as $\arccos (|\boldsymbol{R} \cdot \boldsymbol{B}| /|\boldsymbol{R}||\boldsymbol{B}|)$, so that $\theta_{\mathrm{RB}}$ outside of $90^{\circ}$ appear in the angle range of $\left[0^{\circ}, 90^{\circ}\right]$.

Figure 2 shows that the spectral break position does not change significantly with increasing $\theta_{\mathrm{RB}}$. The independence of $f_{\mathrm{b}}$ on $\theta_{\mathrm{RB}}$ indicates an isotropy of the onset of dissipation and/ or dispersion in kinetic turbulence. The isotropy of $f_{\mathrm{b}}$ is an interesting new result, which makes it remarkable when considering the anisotropy of both power level and spectral index as discovered in previous works (Horbury et al. 2008; Podesta 2009). The anisotropy of the power level and spectral index in our work is similar to the anisotropies in these previous studies. The spectral index in the parallel direction is about -1.9 , and the spectral index in the perpendicular direction is about -1.6 , which indicates the possible existence of critical balance. The integrated power level is also greater in the perpendicular direction than in the parallel direction. Figure 3 further illustrates the angular profile of $f_{\mathrm{b}}$, which stays around $f_{\mathrm{c}}(\sim 0.5 \mathrm{~Hz})$ throughout the whole range of $\theta_{\mathrm{RB}}$. It can be seen that $f_{\mathrm{b}}$ is well below $f_{\mathrm{d}}$ and $f_{\rho}$ and well above $f_{\mathrm{g}}$, with no error bars of $f_{\mathrm{b}}$ crossing the horizontal lines of $f_{\mathrm{d}}, f_{\rho}$, and $f_{\mathrm{g}}$ in Figure 3. The uncertainty of the spectral break position (the length of the error bar) is estimated with the bootstrapping procedure. The bootstrapping method applied here involves three steps: (1) we obtain a new discrete data set for the spectral profile with two random frequency points being taken away from the original data set and fit it with the dual-power-law function, providing the spectral break position at the maximum curvature; (2) we repeat the first step 199 times with different points removed from the data set; (3) we calculate the standard deviation based on the 200 spectral break positions obtained in the first and second steps. The uncertainties of the characteristic frequencies are also illustrated in Figure 3. The characteristic frequencies' uncertainties are estimated from error propagation of the physical variables listed in the upper-right corner of Figure 1. We see that the spectral break frequencies at various angles fall within the uncertainty range of the cyclotronresonance frequency.

As to the systematic uncertainty of the break position, we consider an alternative definition by adopting the maximum of the second derivative $\left(\frac{d^{2} \lg \text { PSD }}{d \lg f^{2}}\right)$ to locate the spectral break. The 


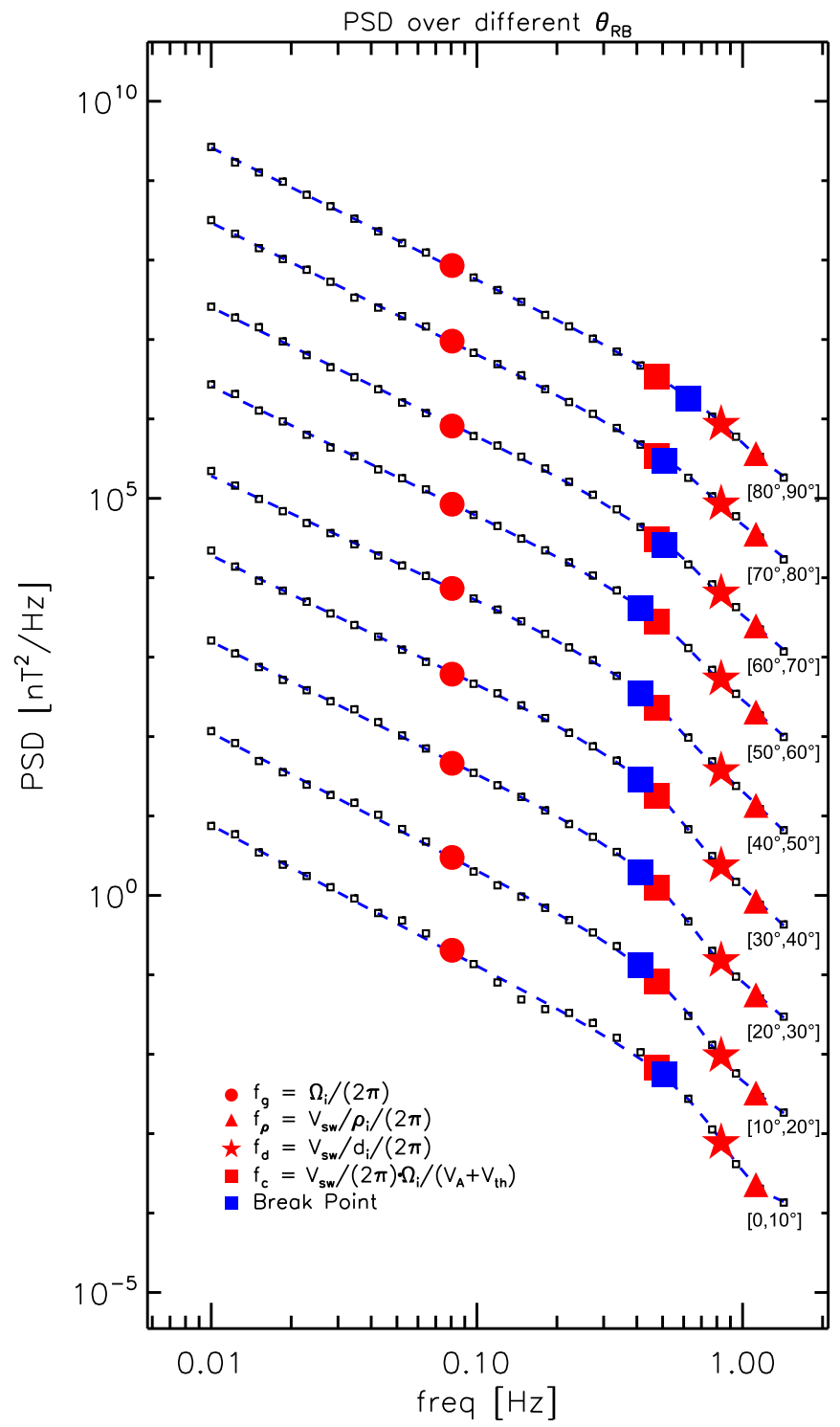

Figure 2. PSDs in different angle intervals of $\theta_{\mathrm{RB}}$ for the same case as in Figure 1. The lowest line represents the PSD in the interval $\theta_{\mathrm{RB}}=\left[0^{\circ}, 10^{\circ}\right]$ and the top line represents the PSD in the interval $\theta_{\mathrm{RB}}=\left[80^{\circ}, 90^{\circ}\right]$. The amplitudes are multiplied with an offset of 10 for each $\theta_{\mathrm{RB}}$ to separate the spectra at different $\theta_{\mathrm{RB}}$. We find that the spectral break position $f_{\mathrm{b}}$ is almost independent of $\theta_{\mathrm{RB}}$.

newly located break positions agree well with the break positions determined with our original method.

As complementary evidence, we show the local slope of the spectra as functions of the frequency for different angles in Figure 4 . We identify the part of the spectrum with a slope less than 2.0 as the inertial range (blue part in Figure 4), and the part with a slope greater than 2.5 as the dissipation range (color coded with yellow and red). The transition at which the contours of the slope distribution are most concentrated, indicating a rapid change of the slope value over a short frequency range, represents the spectral break position. We find that the break position changes only slightly with angle. Additionally, the absolute value of the slope in the dissipation range increases as the angle increases, with $\alpha>4$ at $\theta_{\mathrm{RB}}$ near $0^{\circ}$ and $\alpha<3$ at $\theta_{\mathrm{RB}}$ near $90^{\circ}$. We note that the secondary increase of the slope at small angle and high frequency may be due to noise.

\section{Statistical Study of the Angular Independence of the Spectral Break Position}

To further check if the finding of the angular independence of spectral break position is a common phenomenon in the fast solar wind, we perform a statistical study of fast streams as measured by the WIND spacecraft between 1995 and 1996. We select fast-stream cases according to the criteria described in the previous section. Twenty cases of high-speed solar wind are selected for further study. For every case, we conduct the same analysis procedure: (1) we obtain the reduced PSDs at different $\theta_{\mathrm{RB}}$; (2) we apply the dual-power-law fitting method to the PSDs with the frequency covering both the inertial and dissipation ranges; (3) we estimate the spectral break position based on the maximum curvature of the fitted dual-power-law profile. In this way, we obtain the spectral break frequency $f_{\mathrm{b}}$ at different $\theta_{\mathrm{RB}}$ for every case.

We then determine the ratios of the frequencies $f_{\rho}, f_{\mathrm{d}}, f_{\mathrm{c}}$, and $f_{\mathrm{g}}$ to the spectral break frequency $\left(f_{\mathrm{b}}\right)$ as functions of $\theta_{\mathrm{RB}}$ and plot them in Figure 5. The mean values of $f_{\rho} / f_{\mathrm{b}}, f_{\mathrm{d}} / f_{\mathrm{b}}, f_{\mathrm{c}} / f_{\mathrm{b}}$, and $f_{\mathrm{g}} / f_{\mathrm{b}}$ as functions of $\theta_{\mathrm{RB}}$ are plotted as a red long-dashed line, a red dotted-dashed line, a red solid line, and a red shortdashed line, respectively. The lower and upper envelopes of these ratios at every $\theta_{\mathrm{RB}}$ are connected and illustrated as areas filled with light blue, light green, light gray, and dark green colors. The reference line for $f_{\mathrm{b}} / f_{\mathrm{b}}$ is located within the envelope area of $f_{\mathrm{c}} / f_{\mathrm{b}}$, yet well below the lower envelopes for both $f_{\rho} / f_{\mathrm{b}}$ and $f_{\mathrm{d}} / f_{\mathrm{b}}$ as well as above the upper envelope for $f_{\mathrm{g}} / f_{\mathrm{b}}$. This statistical study reveals two findings with respect to the spectral break in the fast solar wind: (1) $f_{\mathrm{b}}$ is mostly independent of $\theta_{\mathrm{RB}}$; and (2) $f_{\mathrm{b}}$ is close to $f_{\mathrm{c}}$ throughout the whole range of $\theta_{\mathrm{RB}}$. In the case of intermediate plasma $\beta$ ( $\beta \sim 1)$, the difference of scales for different predictions is of order of unity. In these cases, it is necessary to acquire further evidence as to which mechanism dominates the spectral slope of the PSD.

\section{Summary and Discussion}

\subsection{Summary of the Observational Analysis Results and Their Relation to Existing Theories}

In order to learn if the onset of dissipation and/or dispersion is isotropic at the ion kinetic scale, we have studied the relation between the angle $\left(\theta_{\mathrm{RB}}\right)$ and the position of the spectral break between the inertial and dissipation ranges. We first calculate the reduced PSDs of magnetic field turbulence corresponding to different $\theta_{\mathrm{RB}}$ based on the measurements from the WIND/ MFI in long-lasting fast streams. We qualify the PSD profiles involving both inertial and dissipation ranges by developping a fitting method with a dual-power-law fitting function. The spectral breaks are located at the frequencies of maximum curvature in the fitted profile. The spectral break frequency in the spacecraft reference frame is almost independent of $\theta_{\mathrm{RB}}$. Such independence suggests that the onset of dissipation and/or dispersion is isotropic with respect to the local mean magnetic field direction. This is an interesting and unanticipated result considering the anisotropy of the turbulence power level and scaling index. 


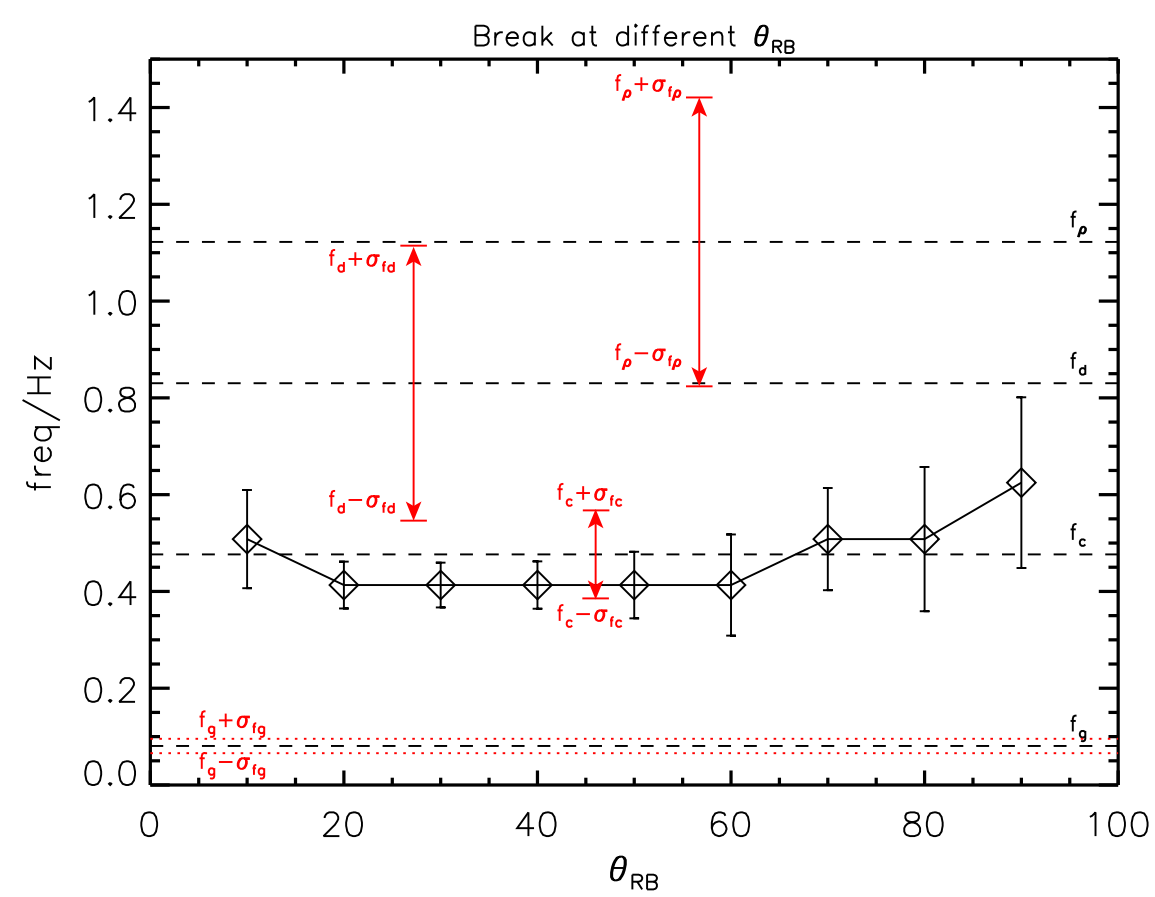

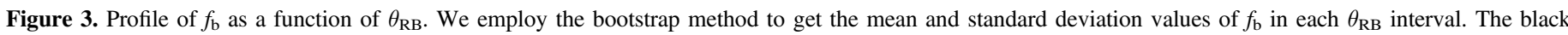

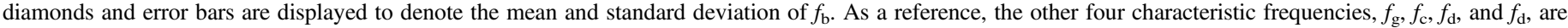
marked as four dashed lines from bottom to top. The standard deviations of these characteristic frequencies are marked as red arrows or red dotted lines.

Furthermore, we find that $f_{\mathrm{b}}$ is consistent with the value of $f_{\mathrm{c}}$ at all angles. Alfvén cyclotron waves in the quasi-parallel direction of propagation are subject to the dissipation through ion-cyclotron resonance. In the reference frame of proton motion with a field-aligned parallel speed of $v_{\text {th }}$, the Doppler shifted wave frequency can approach the proton gyrofrequency, $\omega+k_{\|} V_{\mathrm{th}}=\Omega_{\mathrm{p}}$, where $\Omega_{\mathrm{p}}$ is the proton gyrofrequency. The corresponding $k_{\|}$satisfies the condition of $k_{\|}\left(d_{\mathrm{p}}+\rho_{\mathrm{p}}\right)=1$, which is also reported by Woodham et al. (2018) in their independent work.

However, the dissipation of quasi-parallel Alfvén waves cannot be responsible for the location of the spectral break at a similar scale in the reduced PSD in the quasi-perpendicular direction. For quasi-perpendicular kinetic waves with $k_{\perp} \gg k_{\|}$, the dispersion relations are $\omega^{2}=k_{\|}^{2} V_{\mathrm{A}}^{2} k_{\perp}^{2} \rho_{\mathrm{p}}^{2} /\left[\beta_{\mathrm{p}}+2 /\left(1+T_{\mathrm{p}} / T_{\mathrm{e}}\right)\right]$ for kinetic Alfvén waves, and $\omega^{2}=k_{\|}^{2} V_{\mathrm{A}}^{2} k_{\perp}^{2} d_{\mathrm{p}}^{2}$ for whistler waves, (Chen \& Boldyrev 2017). We note that the normalized terms $k \rho_{\mathrm{p}}$ or $k d_{\mathrm{p}}$ are introduced into their dispersion relation, respectively, playing a role in regulating the dispersion from a linear frequency-towavenumber relation to a nonlinear frequency-to-wavenumber relation. As a result, the electric field disturbance $\delta E$ is proportional to $k_{\perp} \rho_{\mathrm{p}} V_{\mathrm{A}} \delta B$ or $k_{\perp} d_{\mathrm{p}} V_{\mathrm{A}} \delta B$, and the velocity disturbance $\delta V$ scales with $k_{\perp} \rho_{\mathrm{p}} V_{\mathrm{A}} \delta B B_{0}$, which hence changes the eddy turnover time $\tau$ by altering the $\delta V_{\perp}$ fluctuation, $\tau \sim l / \delta V$, from being proportional to $1 / k_{\perp}$ to being proportional to $1 / k_{\perp}^{2}$. In this way, the power-law scaling index for the PSD of $\delta B$ changes from about $-5 / 3$ in the inertial range to about $-7 / 3$ in the kinetic range, causing a spectral break around $k_{\perp} \rho_{\mathrm{p}} \sim 1$ for kinetic Alfvén waves or $k_{\perp} d_{\mathrm{p}} \sim 1$ for oblique whistler waves, respectively. One type of wave mode alone is unable to explain the observed spectral break position. Moreover, the dispersion effect introduced by kinetic Alfvén waves or oblique whistler waves alone is not sufficient to explain the complex physics around the spectral break. The dissipation effect of kinetic waves needs to be taken into account for the spectral profile, e.g., electron and proton Landau damping, transit-time damping, as well as the dissipation by nonlinear stochastic perpendicular heating. The predicted spectral break position as obtained from the dissipation of dispersive kinetic waves in theoretical modeling (Howes et al. 2008; Franci et al. 2016), however, is still not fully consistent with the observed spectral break position. Furthermore, there are small located enhancements in the quasi-parallel PSDs as shown in Figure 2. These enhancements indicate the local injection of energy, which may be due to the local action of instabilities, although we cannot exclude an instrumental effect as their source.

\subsection{An Interpretation: Spatial Scale Estimate for the Onset of the Breakdown of the Magnetic Frozen-in Condition}

At kinetic scales, in contrast to the MHD regime protons, and electrons are successively decoupled from the magnetic field lines. This demagnetization of the plasma at kinetic scales is also responsible for magnetic reconnection. The Generalized Ohm's law provides the framework for further analysis of the breakdown of the frozen-in condition:

$$
\boldsymbol{E}=-\boldsymbol{V}_{\mathrm{p}} \times \boldsymbol{B}+\boldsymbol{J} \times \boldsymbol{B} /(n e)-\boldsymbol{\nabla} \cdot \boldsymbol{P}_{\mathrm{e}} /(n e) .
$$

The Hall term $(\boldsymbol{J} \times \boldsymbol{B} /(n e))$ and the term for the divergence of the electron thermal pressure tensor $\left(-\boldsymbol{\nabla} \cdot \boldsymbol{P}_{\mathrm{e}} /(n e)\right)$ are two important terms to break the magnetic field frozen-in condition. Both whistler wave dynamics and kinetic Alfvén wave dynamics, which are mainly connected with the Hall term and the pressure-tensor-divergence term, respectively, are related to the dissipation region of the reconnection current layer. Therefore, the width of a reconnection current sheet is in principle determined by the ion kinetic scale, e.g., $d_{\mathrm{p}}$ or $/$ and $\rho_{\mathrm{p}}$, although the relation between the current sheet width and the ion kinetic scales has yet to be determined. 


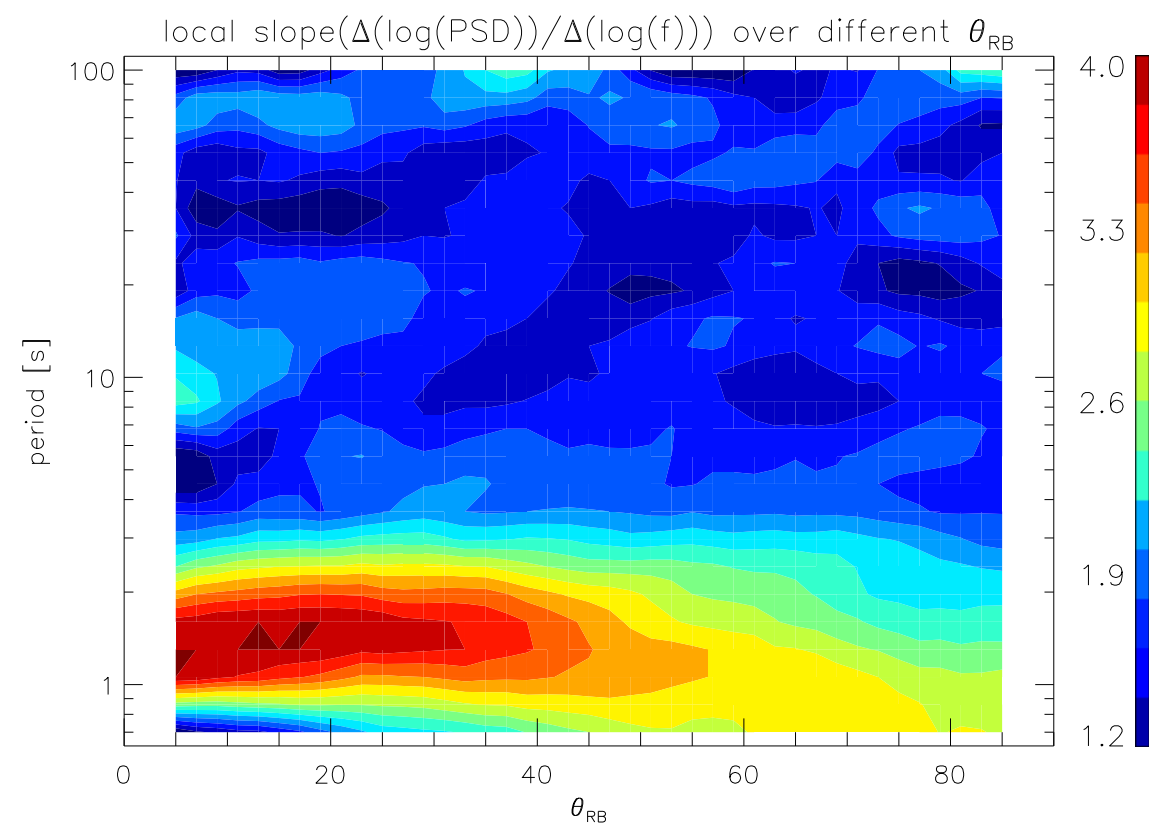

Figure 4. Distribution of absolute local spectral index in the 2D space of $\theta_{\mathrm{RB}}$ and period. The transition of absolute spectral index from less than 2 (blue color) to more than 2.5 (green, yellow, and red colors) occurs roughly around a constant period over the whole range of $\theta_{\mathrm{RB}}$. This suggests an isotropy of the spectral break regardless of the $\theta_{\mathrm{RB}}$ value, although the spectral scaling anisotropy is obvious in the dissipation range below the spectral break.

In some sense, the dissipation of magnetic energy associated with kinetic waves is related to the break down of the magnetic frozen-in condition. On the other hand, the modification of dispersion due to kinetic effects is also related to the decoupling between the fluid species and their individual demagnetization. Therefore, we now investigate the transition between the frozen-in and unfrozen-in states in order to understand the physics of the spectral break, which involves both dissipation and dispersion effects. In Equation (5), if the last two terms on the right-hand side (RHS) dominate over the first term on the RHS, the protons decouple from the electrons and demagnetize. The relation involving the three terms can be approximated as

$$
V B \lesssim \frac{B^{2}}{\mu_{0} n e \Delta}+\frac{T_{\mathrm{e}}}{T_{\mathrm{p}}} \frac{n m_{\mathrm{p}} V_{\mathrm{th}, \mathrm{p}}^{2}}{n e \Delta},
$$

where $V$ is the proton bulk velocity fluctuation at the spatial scale $\Delta$. This requires the spatial scale $\Delta$ in Equation (6) to fulfill the following inequality condition:

$$
\Delta \lesssim \frac{B}{\mu_{0} n e V}+\frac{T_{\mathrm{e}}}{T_{\mathrm{p}}} \frac{m_{\mathrm{p}} V_{\mathrm{th}, \mathrm{p}}^{2}}{e B V}
$$

which can be further grouped into three categories according to the value of the proton plasma beta:

$$
\begin{aligned}
& \Delta \lesssim 2 \pi\left(d_{\mathrm{p}}+\rho_{\mathrm{p}} \sqrt{\beta_{\mathrm{p}}} \frac{T_{\mathrm{e}}}{T_{\mathrm{p}}}\right) \sim 2 \pi d_{\mathrm{p}}, \\
&\left(\beta_{\mathrm{p}} \ll 1, V \sim V_{\mathrm{A}} / 2 \pi \gg V_{\mathrm{th}, \mathrm{p}} / 2 \pi\right), \\
& \Delta \lesssim 2 \pi\left(d_{\mathrm{p}}+\rho_{\mathrm{p}} \frac{T_{\mathrm{e}}}{T_{\mathrm{p}}}\right), \\
& \quad\left(\beta_{\mathrm{p}} \sim 1, V \sim V_{\mathrm{A}} / 2 \pi \sim V_{\text {th, }} / 2 \pi\right),
\end{aligned}
$$

$$
\begin{gathered}
\Delta \lesssim 2 \pi\left(d_{\mathrm{p}} / \sqrt{\beta_{\mathrm{p}}}+\rho_{\mathrm{p}} \frac{T_{\mathrm{e}}}{T_{\mathrm{p}}}\right) \sim 2 \pi \rho_{\mathrm{p}} \frac{T_{\mathrm{e}}}{T_{\mathrm{p}}}, \\
\left(\beta_{\mathrm{p}} \gg 1, V \sim V_{\text {th }, \mathrm{p}} / 2 \pi \gg V_{\mathrm{A}} / 2 \pi\right) .
\end{gathered}
$$

In the situation of extremely low plasma beta, the ratio of average $T_{\mathrm{e}}$ to average $T_{\mathrm{p}}$ is about 10 in the solar wind (Chen et al. 2014). The large value of $T_{\mathrm{e}} / T_{\mathrm{p}}$ causes the $\rho_{\mathrm{s}}$ scale (ion sound gyroradius, $\rho_{\mathrm{s}}=\sqrt{k_{\mathrm{B}} T_{\mathrm{e}} / m_{\mathrm{p}}} / \Omega_{\mathrm{p}}$ ) to be larger than $\rho_{\mathrm{p}}$. However, in the case of low beta, the spectral break is mainly controlled by the ion inertial length $\left(d_{\mathrm{p}}\right)$ rather than $\rho_{\mathrm{s}}$ or $\rho_{\mathrm{p}}$. For the cases with extremely high plasma beta, the ratio $T_{\mathrm{e}} / T_{\mathrm{p}}$ is about 2 on average (Chen et al. 2014), and the scales do not differ significantly. Therefore, the above approximate formulas are comparable (though not fully consistent) with the results illustrated in this work based on intermediate $\beta_{\mathrm{p}}$ and the results for $\beta_{\mathrm{p}} \gg 1$ and $\beta_{\mathrm{p}} \ll 1$ in Chen et al. (2014). Nevertheless, the deviation of $T_{\mathrm{e}} / T_{\mathrm{p}}$ from 1 in the intermediate and high plasma beta cases, still complicate the comparison between observations and theoretical predictions. We note that this is just a rough estimate of the spectral break position by regarding it as the onset of the breakdown of the frozen-in condition and demagnetization, under which condition the dissipation and dispersion of turbulence begin. This idea is essentially similar to the concepts explored in previous works. Here we incorporate both the dispersion and dissipation into a unified description, in order to facilitate the discussion of the spectral break formation.

\subsection{Roles of Dispersion and Dissipation in PSD Deformation, and the Relation to the Breakdown of the Frozen-in Condition in Wavenumber Space}

In a one-dimensional model of the turbulent cascade, the energy transfer rate $\epsilon$ is related to the turbulence amplitude in two ways: (1) through definition based on dimensional analysis, 


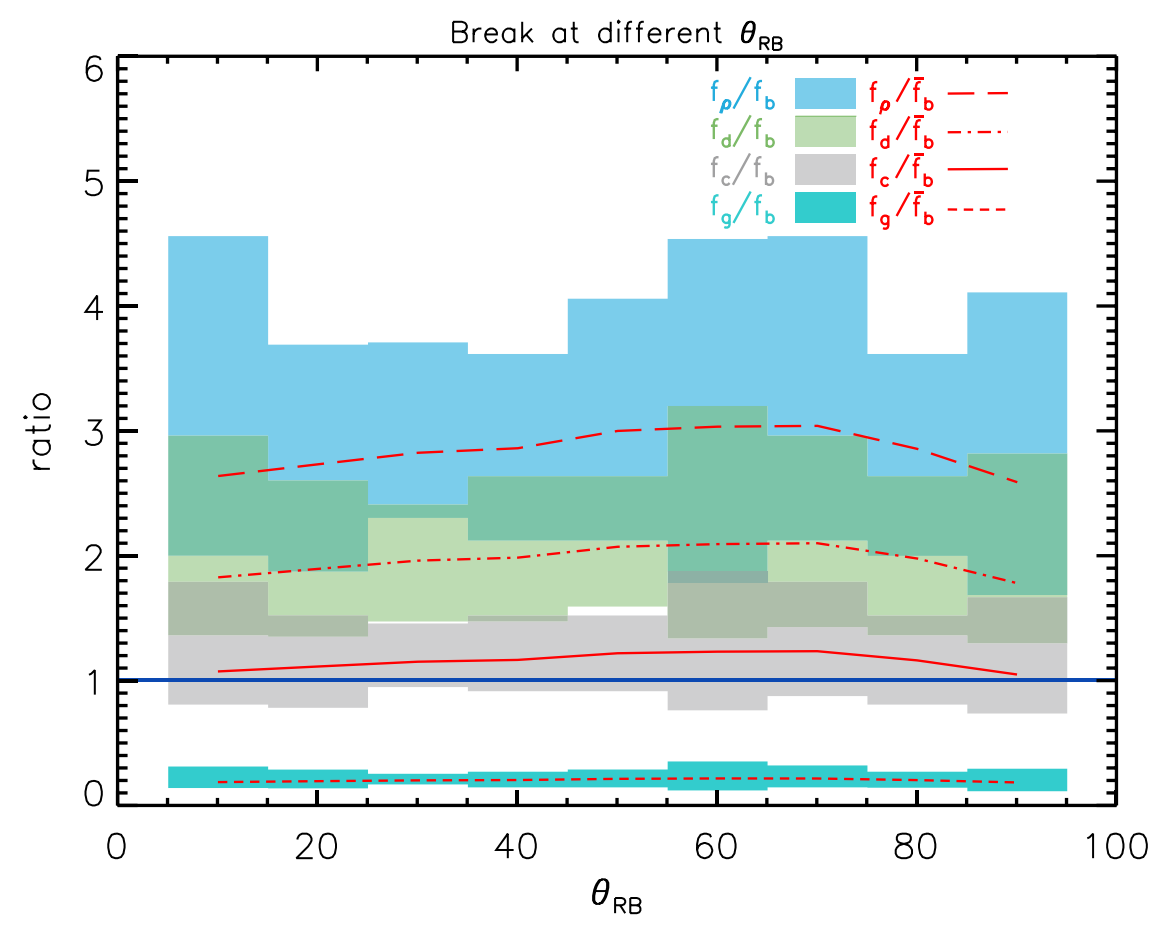

Figure 5. Statistic result of the frequency ratios between the characteristic frequencies and break frequencies in all of the 20 cases under investigation. The colored polygons from top to bottom are bounded by the lower and upper envelops for $f_{\rho} / f_{\mathrm{b}}, f_{\mathrm{d}} / f_{\mathrm{b}}, f_{\mathrm{c}} / f_{\mathrm{b}}$, and $f_{\mathrm{g}} / f_{\mathrm{b}}$. Darker colored areas indicate overlapping areas. Red thin lines of different types denote the mean values of each ratio. The reference level of 1 (red solid straight line) falls in the gray-colored polygon $\left(f_{\mathrm{c}} / f_{\mathrm{b}}\right)$, which suggests that $f_{\mathrm{b}}$ is independent of $\theta_{\mathrm{RB}}$ and the corresponding wavenumber $k$ is determined by $k\left(\rho_{\mathrm{p}}+d_{\mathrm{p}}\right)=1$.

(2) by enforcing an isotropic evolution in wavenumber space:

$$
\epsilon(k)=C_{1}^{-3 / 2} \frac{\delta B(k)^{2}}{1 /(k \delta v(k))},
$$

and

$$
\frac{\partial \delta B(k)^{2}}{\partial t}=-k \frac{\partial \epsilon(k)}{\partial k}-2 \gamma \delta B(k)^{2}+S(k)
$$

where $\gamma$ represents the energy damping rate, $S(k)$ is a source term for the turbulence driving source, and $C_{1}$ is a constant. By assuming $\delta v(k)= \pm \sqrt{R_{\mathrm{A}}^{*}(k)} \delta B(k)$ and then substituting the expression of $\delta B(k)$ with $\epsilon(k)$ into Equation (12), we obtain the following integral formula after some calculations,

$$
\epsilon(k)=\epsilon_{\text {input }} \exp \left\{-\int_{k_{\text {input }}}^{k} 2 C_{1}^{3 / 2} C_{2} \frac{1}{k} \frac{\gamma(k)}{\omega(k)} d k\right\},
$$

where the subscript "input" represents the outer scale of energy input ( $C_{2}$ is also a constant). It can be seen in Equation (13) that, both the dispersion $\omega(k)$ and dissipation $\gamma(k)$ are incorporated into the cascading rate formula, thereby adjusting the value of the cascade rate along with $k$. Finally, the formula for the $\operatorname{PSD}(\delta B(k))$ is written as

$$
\begin{aligned}
& \operatorname{PSD}(\delta B(k)) \sim \frac{\delta B(k)^{2}}{k} \\
& \quad=C_{\mathrm{l}} \epsilon(k)^{2 / 3} k^{-5 / 3} R_{\mathrm{A}}^{*}(k)^{-1 / 3},
\end{aligned}
$$

where the PSD is steeper if $\epsilon(k)$ decreases with $k$ or/and $R_{\mathrm{A}}^{*}$ increases with $k$. According to Equation (13), a decreasing trend of $\epsilon(k)$ is either associated with an increasing trend of $\gamma(k)$ or a decreasing trend of $\omega(k)$. Therefore, in order to understand the PSD variation in $k$, we require the distribution of $\omega(k), \gamma(k)$, and $R_{\mathrm{A}}^{*}$, with the first and third quantities being related to the dispersion and the second term corresponding to the dissipation. We note that Equations (11)-(14) are similar to those by Howes et al. (2008). The variable $R_{\mathrm{A}}^{*}$ can be regarded as the Alfvén ratio in the MHD regime, although it loses its meaning in the kinetic regime, where $R_{\mathrm{A}}^{*}$ is related to $\delta \boldsymbol{V}_{\mathrm{e}}$ rather than $\delta \boldsymbol{V}_{\mathrm{i}}$.

As an example, we study the dispersion, dissipation, and demagnetization (breakdown of frozen-in condition) of Alfvén waves and their counterparts at kinetic scales. The dispersion relation, damping/growth rate, and transport ratios of different variables (e.g., $\delta \boldsymbol{E}, \delta \boldsymbol{B}, \delta \boldsymbol{V}_{\mathrm{i}}, \delta \boldsymbol{V}_{\mathrm{e}}$, and so on) are calculated with linear Vlasov-Maxwell theory, using the numerical code "NHDS" (Verscharen et al. 2016; Verscharen \& Chandran 2018). Figures 6(a) and (c) show the effect from dispersion, which exhibits an increase of $k_{\perp}$. On the other hand, Figure 6(b) shows that the damping rate increases along the $k_{\|}$direction. Both dispersion and dissipation illustrate anisotropic but different patterns in wavenumber space. Figure 6(d) describes the demagnetization of ions $\left(\delta \boldsymbol{E}_{\text {IonFrame }}=\delta \boldsymbol{E}+\delta \boldsymbol{v}_{\text {ion }} \times \boldsymbol{B}\right.$, where $\delta \boldsymbol{E}_{\text {IonFrame }}=0$ for the frozen-in condition). The demagnetization of ions and the decoupling from electrons introduce effects of dispersion and dissipation into the Alfvén waves. Therefore, Figure 6(d) represents the combined effects of dispersion and dissipation, showing a quasi-isotropy (though not exact isotropy) in wavenumber space compared to Figures $6(\mathrm{a}-\mathrm{c})$.

The break was previously expected to occur at a lower frequency in the parallel direction than in the perpendicular direction, based on the anisotropic pattern of turbulence power (Chen et al. 2010). It is interesting that the measurements here are different from these expectations, which calls for a convincing physical explanation for this phenomenon. Looking 

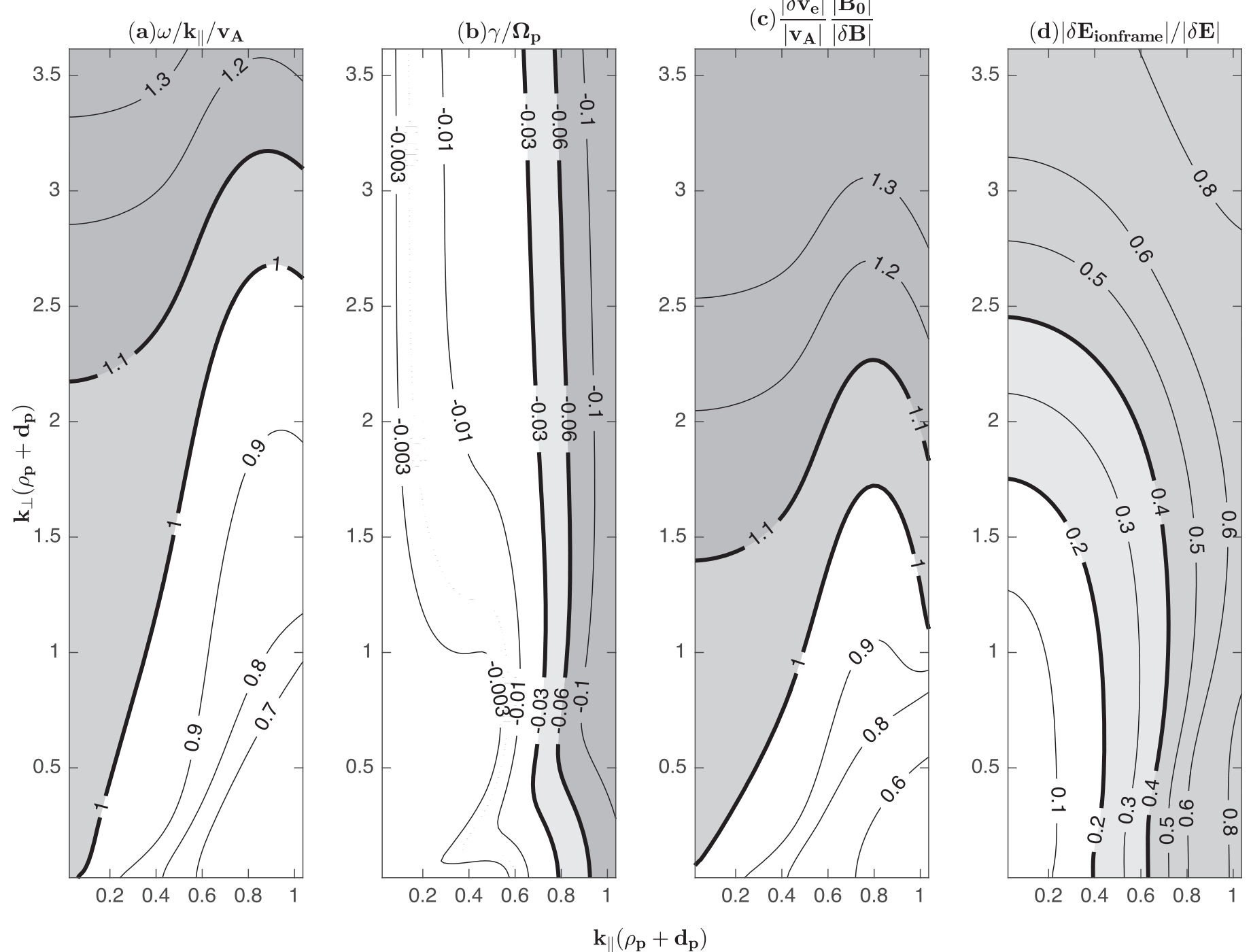

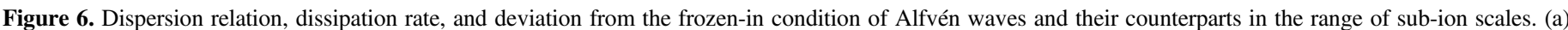

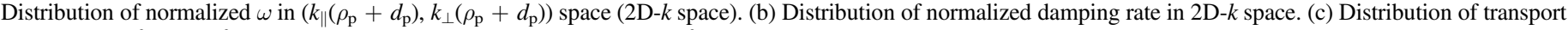

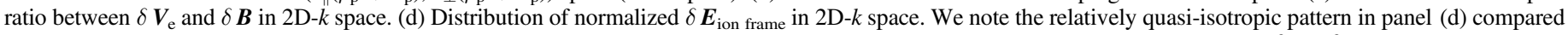
to panels $(\mathrm{a}-\mathrm{c})$. The calculation is conducted in linear Vlasov-Maxwell theory for a plasma only containing protons and electrons $\left(\beta_{\mathrm{p}}=\beta_{e}=2\right)$.

at the distribution of $\delta \boldsymbol{E}_{\text {IonFrame, }}$ in wavenumber space $\left(k_{\|}, k_{\perp}\right)$ is just a preliminary effort. We admit that the occasional existence of a weak enhancement in the parallel spectrum around the break scale complicates the physical scenario for the break formation. We speculate that the weak enhancement may be caused by the injection of local power due to kinetic instabilities. Regarding the breakdown of the frozen-in condition and its role for the spectral break, both dissipation/ damping and instability/growing of kinetic waves are associated with nonzero $\delta \boldsymbol{E}_{\text {IonFrame, and hence result in a spectral }}$ deformation at a similar scale. The enhancement if existent usually appears at angles of less than $20^{\circ}$. For the mostly isotropic pattern beyond $20^{\circ}$, the underlying physical mechanism may be unrelated to the instability-induced local power injection. The 1D equation for the turbulence power as presented above may not be appropriate for the dynamics in the parallel direction, along which the energy transfer may mostly occur through diffusion rather than advection in wavenumber. In the future, it is necessary to investigate and improve the evolution model (advection, diffusion, injection, and dissipation) for the PSD in multidimensional wavenumber space (Cranmer \& Van Ballegooijen 2003), in order to describe both the anisotropy of the power level and the isotropy of the power break position.

For turbulence with most energy located at large oblique angles, $\delta \boldsymbol{E}_{\text {IonFrame }}\left(k_{\|}, k_{\perp}\right)$ would be enhanced at large $\theta_{\mathrm{kB}}$ compared to its values at small $\theta_{\mathrm{kB}}$. As a consequence, $\left|\delta \boldsymbol{E}_{\text {IonFrame }}\left(k_{\|}, k_{\perp}\right)\right| /$ $\left|\delta \boldsymbol{E}_{\text {Fullangle }}(k)\right|$ tends to become more isotropic in turbulence with higher anisotropy than shown in Figure $6(d)$. Therefore, weak anisotropy of $\left|\delta \boldsymbol{E}_{\text {IonFrame }}\left(k_{\|}, k_{\perp}\right)\right| /\left|\delta \boldsymbol{E}_{\text {FullAngle }}(k)\right|$ due to weaker dispersion than dissipation on demagnetization can be compensated and approach isotropy by the dominance of energy at large $\theta_{\mathrm{kB}}$. The contribution of energy anisotropy to the isotropy of the spectral break will be investigated in our future work. Again, we emphasize that more observational and theoretical studies are needed to 
understand the physics responsible for the angle-independent position of the spectral break, which is close to the scale corresponding to $k\left(d_{\mathrm{p}}+\rho_{\mathrm{p}}\right)$ near $1 \mathrm{au}$.

The authors thank the WIND MFI team and 3DP team for providing the magnetic field and the thermal proton data. This work at Peking University is supported by NSFC under contracts 41574168, 41231069, 41274172, 41474148, and 41421003. J.S.H. is also supported by the National Young Talent Program of China. D. Verscharen is supported by an STFC Ernest Rutherford Fellowship. The authors express their gratitude to C. H. K. Chen for his helpful comments on this work.

\section{ORCID iDs}

Jiansen He (ib https://orcid.org/0000-0001-8179-417X

Daniel Verscharen (ib https://orcid.org/0000-0002-0497-1096

Linghua Wang (iD https://orcid.org/0000-0001-7309-4325

\section{References}

Alexandrova, O., Lacombe, C., Mangeney, A., Grappin, R., \& Maksimovic, M. 2012, ApJ, 760, 121

Alexandrova, O., Saur, J., Lacombe, C., et al. 2009, PhRvL, 103, 165003

Bale, S. D., Kellogg, P. J., Mozer, F. S., Horbury, T. S., \& Reme, H. 2005 , PhRvL, 94, 215002

Bieber, J. W., Wanner, W., \& Matthaeus, W. H. 1996, JGR, 101, 2511

Biskamp, D., Schwarz, E., \& Drake, J. F. 1996, PhRvL, 76, 1264

Biskamp, D., Schwarz, E., Zeiler, A., Celani, A., \& Drake, J. F. 1999, PhPl, 6, 751

Boldyrev, S., Chen, C. H., Xia, Q., \& Zhdankin, V. 2015, ApJ, 806, 238

Bourouaine, S., Alexandrova, O., Marsch, E., \& Maksimovic, M. 2012, ApJ, 749, 102

Bruno, R., \& Carbone, V. 2013, LRSP, 10 doi:10.12942/1rsp-2013-2

Bruno, R., \& Trenchi, L. 2014, ApJL, 787, L24

Cerri, S. S., Califano, F., Jenko, F., Told, D., \& Rincon, F. 2016, ApJL, 822, L12

Chen, C., Horbury, T., Schekochihin, A., et al. 2010, PhRvL, 104, 255002

Chen, C. H. K., \& Boldyrev, S. 2017, ApJ, 842, 122

Chen, C. H. K., Leung, L., Boldyrev, S., Maruca, B. A., \& Bale, S. D. 2014, GeoRL, 41, 8081

Cranmer, S. R., \& Van Ballegooijen, A. 2003, ApJ, 594, 573
Franci, L., Landi, S., Matteini, L., Verdini, A., \& Hellinger, P. 2016, ApJ, 833, 91

Galtier, S. 2006, JPIPh, 72, 721

Galtier, S., \& Bhattacharjee, A. 2003, PhPl, 10, 3065

Goldreich, P., \& Sridhar, S. 1995, ApJ, 438, 763

Hadid, L. Z., Sahraoui, F., \& Galtier, S. 2017, ApJ, 838, 9

He, J., Marsch, E., Tu, C., Yao, S., \& Tian, H. 2011, ApJ, 731, 85

He, J., Tu, C., Marsch, E., Bourouaine, S., \& Pei, Z. 2013, ApJ, 773, 72

He, J., Tu, C., Marsch, E., \& Yao, S. 2012a, ApJL, 745, L8

He, J., Tu, C., Marsch, E., \& Yao, S. 2012b, ApJ, 749, 86

Horbury, T. S., Forman, M., \& Oughton, S. 2008, PhRvL, 101, 175005

Howes, G. G., Cowley, S. C., Dorland, W., et al. 2008, JGRA, 113, A05103

Kiyani, K. H., Osman, K. T., \& Chapman, S. C. 2015, RSPTA, 373, 20140155

Leamon, R. J., Smith, C. W., Ness, N. F., Matthaeus, W. H., \& Wong, H. K. 1998, JGR, 103, 4775

Lepping, R. P., Acna, M. H., Burlaga, L. F., et al. 1995, SSRv, 71, 207

Li, H., Gary, S. P., \& Stawicki, O. 2001, GeoRL, 28, 1347

Lin, R. P., Anderson, K. A., Ashford, S., et al. 1995, SSRv, 71, 125

Luo, Q. Y., \& Wu, D. J. 2010, ApJL, 714, L138

MacBride, B. T., Smith, C. W., \& Forman, M. A. 2008, ApJ, 679, 1644

Matteini, L., Alexandrova, O., Chen, C. H. K., \& Lacombe, C. 2017, MNRAS, 466, 945

Matthaeus, W. H., Goldstein, M. L., \& Roberts, D. A. 1990, JGR, 95, 20673

Narita, Y., \& Gary, S. P. 2010, AnGeo, 28, 597

Narita, Y., Glassmeier, K.-H., Goldstein, M. L., Motschmann, U., \& Sahraoui, F. 2011, AnGeo, 29, 1731

Perri, S., Carbone, V., \& Veltri, P. 2010, ApJL, 725, L52

Podesta, J. J. 2009, ApJ, 698, 986

Roberts, O. W., \& Li, X. 2015, ApJ, 802, 1

Sahraoui, F., Goldstein, M. L., Belmont, G., Canu, P., \& Rezeau, L. 2010, PhRvL, 105, 131101

Sahraoui, F., Goldstein, M. L., Robert, P., \& Khotyaintsev, Y. V. 2009, PhRvL, 102, 231102

Schekochihin, A. A., Cowley, S. C., Dorland, W., et al. 2009, ApJS, 182, 310

Smith, C. W., Vasquez, B. J., \& Hollweg, J. V. 2012, ApJ, 745, 8

Telloni, D., Bruno, R., D’Amicis, R., Pietropaolo, E., \& Carbone, V. 2012, ApJ, 751, 19

Torrence, C., \& Compo, G. P. 1998, BAMS, 79, 61

Tu, C. Y., \& Marsch, E. 1995, SSRv, 73, 1

Verscharen, D., Chandran, B. D., Klein, K. G., \& Quataert, E. 2016, ApJ, 831,128

Verscharen, D., \& Chandran, B. D. G. 2018, RNAA, 2, 13

Wang, X., Tu, C.-Y., He, J.-S., \& Wang, L.-H. 2018, JGRA, 123, 68

Woodham, L. D., Wicks, R. T., Verscharen, D., \& Owen, C. J. 2018, ApJ, 856, 49

Zhao, J. S., Voitenko, Y. M., Wu, D. J., \& Yu, M. Y. 2016, JGRA, 121, 5 